\title{
USER ADAPTATION IN E-LEARNING ENVIRONMENTS
}

\author{
Paraskevi Tzouveli \\ Image, Video and Multimedia Systems Laboratory \\ School of Electrical and Computer Engineering \\ National Technical University of Athens \\ tpar@image.ntua.gr
}

\author{
Stefanos Kollias Member IEEE \\ Image, Video and Multimedia Systems Laboratory \\ School of Electrical and Computer Engineering \\ National Technical University of Athens \\ stefanos@cs.ntua.gr
}

\begin{abstract}
In this paper we present a distance-learning scheme that performs dynamic user evaluation in order to allow training of users adaptable to their needs. The appropriate learner profile is selected on-line, influencing the level and the contents of the offered courses while behavioral evaluation affects the pace of training and the way information is presented to the user. Learner profiles are created and continuously updated through an e-survey tool that is integrated into the system. In its current form the presented system aims at increasing the familiarity with ICT of teachers working in the Special Education sector.
\end{abstract}

Key words: user adaptable system, e-learning, e-survey, ICT level estimation, ICT statistics 


\section{INTRODUCTION}

The impact of Information and Communication Technologies (ICT) has become more and more evident in learning and teaching at all levels of education. An ICT-based distance learning system consists of a set of electronic tutorials, information and tools which provide their users with enriched capability of accessing relative information.

However, existing e-learning systems, usually being of a self study nature, are not suitable for learners that do not already possess some knowledge about the topic that interests them. For such learners, the interaction with a real life teacher remains indispensable; automated on-line systems that are able to offer learning material modulated to the learning needs of every individual are, therefore, of great importance in modern ICTbased environment.

In this paper we make an attempt to narrow this difficulty. Specifically, we use the IEEE Learning Technology Systems Architecture [1] and develop an e-learning system that is able to adapt its operation to the learning needs of its users.

Our approach is based on the usage of electronic questionnaires (equestionnaires) that are designed by a group of experts and aim to detect the user's cognitive level and learning preferences prior to the learning experience. Through analysis of their responses to the e-questionnaires, users can be assigned to corresponding profiles, and be consequently provided with learning material that best matches their needs.

As a case study, the proposed system in this paper has been utilized in the framework of "SPERO: A TeleInformatics System for Continuous Collection, Processing, Diffusion of Material for Teacher Training in Special Education", a European project [2] for the tuition of teachers, mainly of the field of special education, in Information and Computer Technologies (ICT) related topics. For this purpose, information concerning the ICT level of teachers has been collected through a pan-European survey; analysis of it has been performed and the main conclusions have been included in the initial form of an e-questionnaire.

The structure of the paper is as follows: in Section 2 we briefly present the general architecture of our system. In Section 3 we explain the adaptation of IEEE LTSA model and its usage in our system. Continuing, in section 4, we describe the design and structure of the e-questionnaires. Moreover, in section 5 , we explain how an e-survey can be conducted. Section 6 describes how the e-questionnaires may be utilized towards the generation of user adaptable e-learning systems. Sections 7 and 8 illustrate the mechanism of the selection of e-courses and present a test bed environment for e-courses. In Section 9 we list our concluding remarks and future work plans. 


\section{SYSTEM'S ARCHITECTURE}

The proposed system is an e-learning system which can be adapted to the user's profile and interests. The main goal of this system is the characterization of the ICT level of users and its matching to the appropriate learning material and e-courses that will help them to improve their ICT level. In our study case, users are teachers who are called to answer a set of e-questionnaires. After completing of the questionnaires, teachers' profiles will be adapted to the system and then they will receive a set of e-lessons for improving their ICT level. Our aim is to propose a system architecture that can form a general architecture for an e-learning schema.

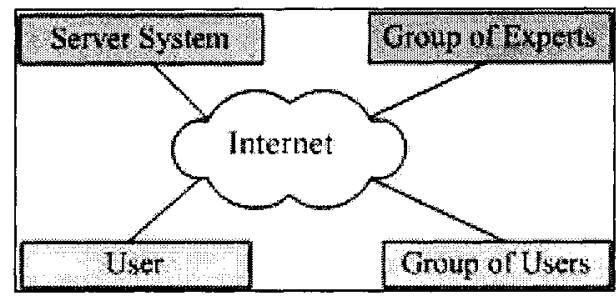

Figure 1. System architecture

The components that compose the general architecture (Figure 1) of the proposed system are the following:

Group of Users: in our study case, the group of users includes teachers who are working in any sector of education (general or special education). In an overview of our system's architecture, learners can include any student, teacher, parent, researcher, or employee. For this reason an adaptation of equestionnaires to the users' needs is necessary.

Group of Experts: includes a variety of people, such as teachers, teachers of the Special Education sector, experts in e-learning, data analysts, psychologists and software engineers.

Server's System: includes the hardware and software that is suitable for the activation of a web-server. This system also includes web applications, such as e-questionnaires, e-survey and e-courses together with an RDBMS system.

Interaction between the components of the system can be achieved through the Internet using the proper equipment. To implement this architecture, the following steps have been followed.

Firstly, the group of experts investigated and analyzed the needs of the group of users. Then the experts defined the learning resources and design a group of questionnaires from which the users' profiles for this particular group of users can be extracted. 
Subsequently, the software engineers have created an e-questionnaire database that is being updated with entries (texts of the questionnaires). The proper software has been developed for automatic retrieval and presentation of the questionnaires from the e-questionnaire database. The proposed software supports multilingual questionnaires.

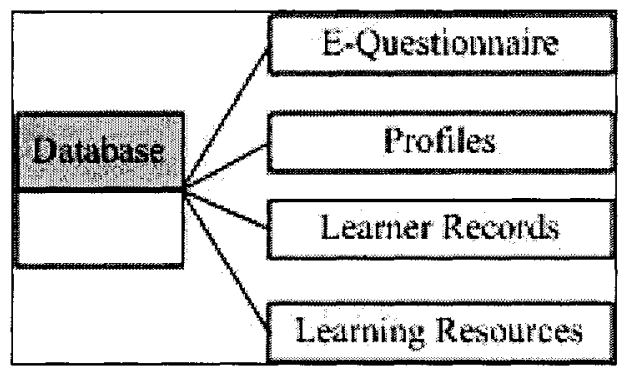

Figure 2. Database content

Additionally, software for automatic survey conduction based on users' answers has been developed. Conclusions derived out of the e-surveys, as well as feedback from the use of the e-questionnaires, are fed back to the equestionnaires. User's profile categorizations are automatically extracted by the system, which takes as inputs the answers of users stored in user records. More than one learning resources (e-courses, multimedia databases including educational TV programmes, newspapers' files, TV files) are selected by the experts, for each distinct collaborative user profile.

\section{A REFINED IEEE E-LEARNING MODEL}

The proposed system's architecture is based on the IEEE Reference Model [1]. This standard specifies a high level architecture for e-learning systems and describes the conceptual design and the generic components of these systems. The LTSA system is separated into three main categories (Figure 3): the Processes (oval shapes), the Store (orthogonal parallelograms) and the Flows (vectors).

The 'processes' category contains entities like learner, evaluation, coach and delivery. The 'stores' category includes databases in which learner records and learning resources have been stored. The interaction between learner and system is controlled by 'flows' which can be the learning preferences, the behaviour, the assessment information, the performance and the preference information, the query, the catalog info, the locator, the learning content and the multimedia resources. 


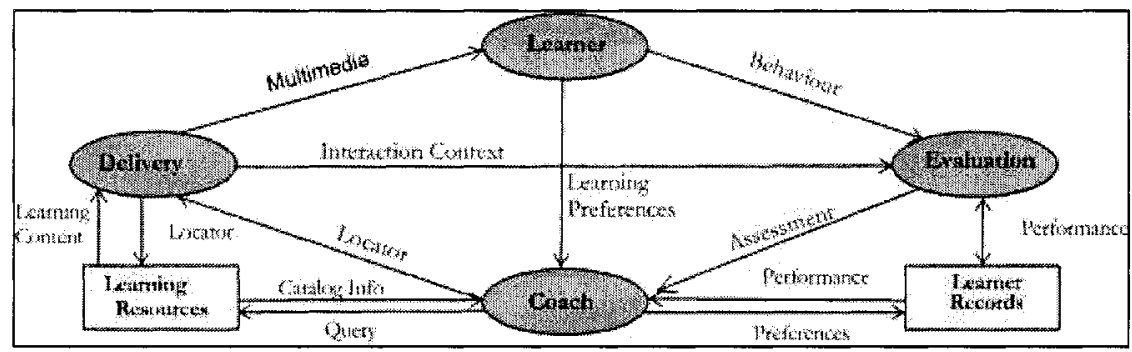

Figure 3. LTSA System Components

The main entity of the IEEE LTSA system is the Learner. A learner can be a single learner or a group of learners with different needs. Firstly, the learners receive an interactive framework, e.g. multimedia presentation; while the presentation is shown to the learners, their behaviours (e.g. responses to questions) are observed. This feedback brings data to the learner in order to enhance the learning profile of the learner.

The learning preferences are combined with the coach process, based on the language and cultural adaptation, as well as accessibility for people with physical limitations. The behaviour component, on the other hand, provides information about the learner's activities; this information (as keyboard clicks, mouse clicks, etc) is recorded in real time and used to evaluate the results of learning.

The evaluation process takes as input the learner entity's observable behaviour; the interaction context is the data flow from the delivery component to the evaluation component that may provide the necessary information for interpreting the information contained in the behavioural data flow. The evaluation component produces assessment information which is sent to the coach and creates performance information that is stored in the learner records. Performance information may come from both the evaluation process (e.g., grades on lessons) and the coach (e.g., certifications). The coach may receive performance information from the learner records at any time. Performance information, such as assessment information and certifications, as well as preferences may be stored in the learner records by the coach.

Based on this information, the coach generates queries and forwards them to the learning resources store, in order to request learning materials that are appropriate for each learner. The learning resources store is a database that represents knowledge, information, and other resources used in the learning experiences. It may be represented as a collection of presentations, tutorials, experiments, lessons, etc. The learning resources store replies to the coach with catalogue info, i.e. with a list of locators that 
match the search query. These may be used by the delivery process to retrieve learning content.

Finally, the delivery process transforms information obtained via learning content into a presentation, which is transferred to the learner entity via a multimedia stream. In the LTSA Draft Standard, a system's ability to adapt its operation to the learner is not obvious; although an evaluation process exists, the way via which this process can be utilized to allow for learner adapted operation is not clear.

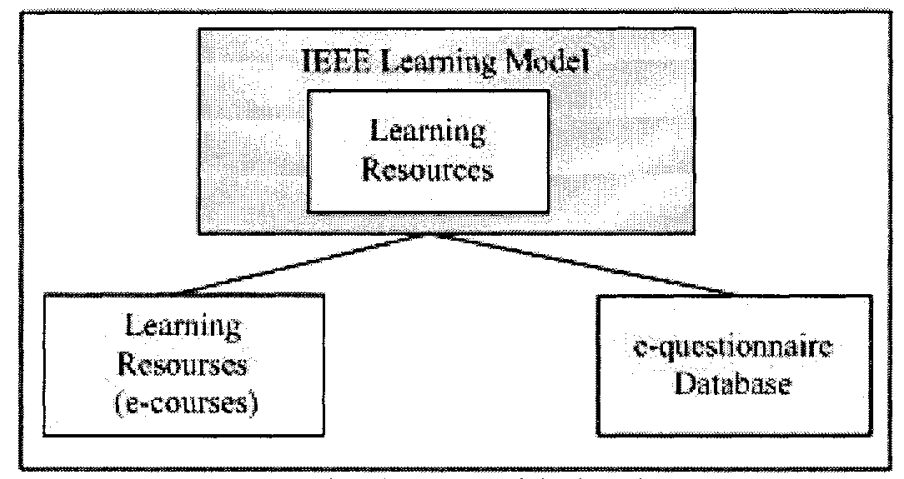

Figure4. Proposed replacement of the learning resources.

For this reason we have enhanced the learning resources component, which stores only representations of knowledge, with the e-questionnaire helping to specific and adapt users' profile within the e-learning system.

In our extension of the LTSA Standard, the learner has been replaced by users like the teacher from a school unit or a group of teachers and the coach has been interchanged with an e-teacher. In order to achieve our scope, we have developed an e-questionnaire through which users' profiles can be extracted, based on users' answers and behaviors. An analytic description of the structure of the e-questionnaire is shown in section 3. This equestionnaire is stored in an e-questionnaire database which is an extension of the learning resources shown in Figure 4.

The learning resources contain data like e-courses, presentations, multimedia data that a user can access after answering the e-questionnaire. So, when users access the system for the first time, they receive an equestionnaire via the delivery process which they are asked to answer. Then, their answers are stored as a record in the e-questionnaire users' answers records. These records can be analyzed and statistics be extracted in the form of reports for a variety of topics regarding e-learning and ICT knowledge. These reports refer to the e-surveys which are described in section 4.

Another refinement that we have made to the LTSA Standard is the extension of the evaluation entity to a re-evaluation entity which extracts 
users' profiles and conducts surveys (Figure 5). The answers to the questionnaire are used for estimating user's profile (for new users) or adapting existing user profiles. Therefore, users are categorized according to their own profile and own other general profile that the database contains. More information about this procedure is given in section 6 .

The behavior system component and the answers to the questionnaire convey the user's component to the evaluation module. It provides "raw" information about users' activities, recorded in real time and used to evaluate the given answers or the performance in courses. User's observable behaviors are inputs to the re-evaluation process. This process produces assessment information (e.g., users' ICT level or grades of users' e-courses) and sends this information to the e-teacher when he asks for it. The reevaluation process creates performance information that is stored in each user's records. In fact, performance information comes from both the reevaluation process (e.g, answers to the questionnaire, grades on lessons) and e-teacher (e.g. obtained certifications).

Users' records hold information about the past (e.g., historical learner records), the present (e.g., current assessments for suspending and resuming sessions) and the future (e.g., pedagogy, learner, or employer objectives). The e-teacher could request and receive performance information from the users' records and also store any users' assessment information and certifications.

The proposed system provides information about the user's current state, which is used by the e-teacher to determine optimal learning experiences or to generate statistics. The e-teacher receives the current assessment information from the re-evaluation process and performance information and supports the decision-making process for choosing next learning experiences.

Based on the current assessment information and historical performance information, the e-teacher sends queries to the learning resources so as to search for learning content that is appropriate for the user. The queries specify search criteria based on, in part, learning preferences, assessment information, and performance and preference information. 


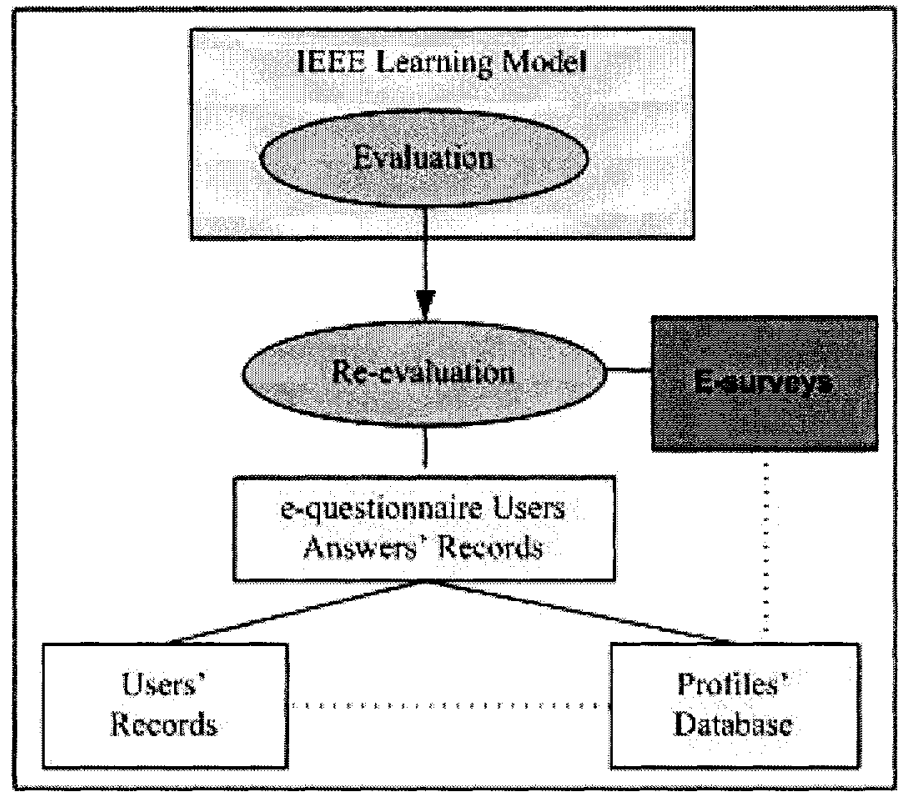

Figure 2. Proposed replacement of the evaluation entity.

The learning resources component stores courses, presentations, tutorials laboratory experiments, and other learning material as resources for learning experiences. The learning resources can be searched by queries. The matched information is returned as catalogue info. The locators are extracted from the catalogue info and can be used by the delivery process to retrieve learning content.

The learning content data flow is a coded representation of material that helps to create the e-teacher as well as to suggest and deliver the learning experience. In fact, the learning content is identified by the locator, retrieved by the learning resources, and transformed by the delivery system into an interactive multimedia learning experience.

The delivery process transforms information obtained via learning content into a presentation, which is transferred to the user. The presentation can be static, interactive, and collaborative or involve experiments and discovery.

The delivery process receives locators from the e-teacher and retrieves learning content from the learning resources. The delivery process transforms the learning content into an e-questionnaire or e-courses for the user. Within an actual system implementation, the delivery process is combined with the re-evaluation process to achieve the tight coupling necessary for responsive, interactive learning experiences. 


\section{DESIGN AND STRUCTURE OF QUESTIONNAIRES}

The proposed questionnaires [5] are developed in the framework of SPERO (Teleinformatics system for continuous collection, processing, diffusion of material for teacher training in special education) [2], a "Leonardo da Vinci" European Programme project.

Firstly, the group of SPERO experts conducted a detailed research in a number of European schools in order to detect a variety of aspects, such as a) the identity and role of teachers in the ICT era; this role is expected to respond to the increasing needs of the current school within the modern social environment, b) the identity of organizations providing teacher training programmes, e.g., universities acting as research and training centers, c) the character of specialized/non specialized training programmes for teachers, d) the attitude of teachers with regard to the use of innovative methods and 'tools', i.e. computers, Internet and software in teaching special education students e) the mechanisms with which European countries train teachers and evaluate training programmes and f) the awareness of teachers with regard to self-evaluation, self-training and life long learning possibilities.

Then, the SPERO users' requirements were analyzed and the educational context was presented to them, especially focusing on Special Education Needs (different levels of education, type of schools, infrastructure of schools, specific national policies) and on the different methods which are used to assess teachers (evaluation procedures). Furthermore, the systematic collection of users' requirements demonstrated the different teacher categories, the educational background of teachers (languages, level of ICT literacy, seminars, training) and the procedures, by which, teachers become qualified in ICT. Following the elaboration of the collective users' requirements, a questionnaire have been designed by the experts. In this questionnaire an explicit segregation of the School and Teacher Profile seem to be essential. So, two separate questionnaires have been generated in order to receive the relevant information. The first group of questionnaires contains questions for school units like the school unit description, students and teachers' population, special educational needs and training programmes for teachers or students from government services, European Union or other organization.

The other group contains questions for teachers like their Information and Computer Technologies (ICT) background. The questions, which are addressed to the teachers, are intended to select information about their professional development, evaluation of educational approach and ICT, their 
familiarization with information technologies and the pedagogical utilization of new technologies.

After the illustration of SPERO questionnaires, each expert for each participating European country has translated the questionnaires in his language and these translations have been stored in the e-questionnaires database. This group of questionnaires can be accessed through the web server [5]. This design can be extended in a general framework of equestionnaires which can vary with the educational needs of the users.

Another approach could lead to a questionnaire design that can cover the needs of a user who wants to search for a particular object in a (multimedia) database.

\section{CONDUCTING E-SURVEYS}

The user answers' records constitute the data for which e-survey is conducted [6]. For this scope, the statistic analysts of the group of experts have analyzed the answers to each question. Moreover the statistical analysis for each country has been performed based on user answers' records through the internet.

The questionnaires fulfil certain requirements in order to ensure that meaningful results are achieved. The data analysts have specified these requirements when designing the e-questionnaires. What each questionnaire is intended to measure has been assigned and assured to be unambiguous and appropriate.

There are various types of validation which we have taken under consideration, such as that data should be complete and cover a broad part of the population for which this survey is conducted. Furthermore, the outcome must be able to be predicted from data, thus sample must be sufficient (10 per cent from SEN school and teachers from participating European countries).

After completion of the collection of users' answers, surveys are conducted. The results of these surveys are the proportions of users' answers per question or the proportions of the users' answers per combinations of questions. In addition, we extract results of combinations of questions and users' profiles. These surveys can be accessed via internet in any language of the participating countries [6]. 


\section{PROFILE EXTRACTION}

The personalization module which extracts the users' profiles, keeps a record of their profiles, containing usage history and user preferences [4]. The user profiles that have been defined by the experts are stored in the profile database. Each one of these describes, in addition to the characteristics of the users it describes, their learning needs and preferences. The answers to the questionnaire are used to select the profile of a new user by finding the existing user profile that best matches the current learner's ICT level of knowledge and peculiarities.

Thus, once a user is assigned to a user profile, the e-teacher uses this information in order to locate in the learning resources store the learning materials that best match his/her profile. A new user profile can be created or existing ones can be adapted based on the statistics of the e-questionnaire database. New user profiles or adapted versions of them are then stored in the Profile Database.

Whenever an already registered user accesses the systems, its profile is restored from the Database Profile. Change of the profile of a user is performed based on the progress of its training. New user profiles are created, based on statistics of the e-survey and are stored in the profiles' database. When users access the proposed system automatically their profiles are recalled from the profiles' database. Any users' level modulation is added to the profiles' database.

\section{E-COURSES: MECHANISM FOR THEIR SELECTION}

SEN teachers will be able to take e-courses in ICT as soon as they complete the e-questionnaire. The SPERO group of experts do not create ecourse for validating the user profile estimation and adaptation based on the questionnaire answers. Instead of this, a list of available e-courses in the SPERO participant countries or elsewhere, are rated according to the required ICT knowledge.

After filling the e-questionnaire, SEN teachers are transferred to the appropriate e-course (if they are willing to attend such a course). The appropriateness of the selected e-courses are estimated by the answers given in the e-questionnaire. A need to rate existing e-courses according to the required level of ICT knowledge is, therefore, necessary. Rating e-courses takes into account the available SPERO user profiles. It is valuable to link several e-courses to each of the SPERO user profiles. Therefore, e-course are rated so as to correspond to an ICT level. Users receive an e-course and their 
behavior is observed. In order to estimate whether the suggested e-course was the one that covers the level of ICT knowledge as well as the requirements of the user, a kind of selection's evaluation is required.

For this evaluation of e-course selection, the behavior of the user when attending the course is recorded, framed in the appropriate context and behavior information is extracted and stored in the user's profile.

\section{TEST BED ENVIRONMENT}

The SPERO total system is a learning portal. When users visit this site, they are asked to answer the e-questionnaire. Automatically, after completion of the e-questionnaires, the user profile is established and stored. Subsequently, a registration form is appears in order to declare users' login name and password for SPERO site. Next, users can access the learning SPERO portal. The main menu of the SPERO portal contains links to the following sectors:

- Courses Catalogue: It contains one or more courses which users may take, according to their ICT level which is automatically extracted from the answer to the questionnaire.

- Announcement : The Announcement Service provides a board where topics about e-courses is published.

- Search: The Search Service provides users with the environment to retrieve specific information existing in the SPERO site, e.g. members, school units, e-lessons.

- E-mail : Users can send or receive e-mails through the SPERO site.

- Upload file: Users have their own personal space where they store their own material to which other users may have access to.

- Help: Its primary purpose is to accommodate HTML pages describing the usage and the tasks of SPERO menu choices.

In order to improve the ICT level of learners, different e-courses can be delivered to them according to their ICT level. Indicatively, e-courses with increasing difficulty include some of the following topics:

- Level 1 : Introduction to Information's Technologies (definition of data, bit, byte, presentation of hardware components, presentation of type of software)

- Level 2: Presentation and usage of operating systems as Microsoft Windows

- Level 3 : Usage of Microsoft Office

- Level 4 : Usage of Internet (Browser, Search Machines, E-mail) 


\section{CONCLUSION}

Among the research community dealing with personalized learning and intelligent learning applications, it becomes clear that the results to be obtained from any kind of electronic questionnaires or surveys will not have the desired effectiveness, unless major focus is given to the individual user profiling based on their history and preferences and the corresponding user behavior.

In this paper we have presented how e-questionnaires, designed for conducting surveys about the ICT level of knowledge of teachers who work in the special education sector, can be used in the framework of a dynamic distance learning scheme in order to achieve user adaptive learning facilities.

\section{ACKNOWLEDGMENT}

This work has been partially funded by the "SPERO: TeleInformatics System for Continuous Collection, Processing, Diffusion of Material for Teacher Training in Special Education" a European project in the "Leonardo Da Vinci" Programme, under "Theme 5: E-Learning".

\section{REFERENCES}

[1] IEEE P1484.1 Draft Standard for Learning Technology- Learning Technology Systems Architecture (LTSA) (http://ieee.ltsc.org/wg1).

[2] SPERO A TeleInformatics System for Continuous Collection, Processing, Diffusion of Material for Teacher Training in Special Education (http://www.image.ntua.gr/spero)

[3] Tzouveli P.,Tsapatsoulis N., Kollias S., Michaelidou M. "On the development of user adapted e-learning schemes for teachers", Proceedings of the 3rd International Conference of New Horizons in Industry and Education, Santorini, Greece, August 2003

[4] Mylonas P., Tzouveli P., Kollias S. "Towards a profiling based e-learning scheme for teachers", ICALT, , August 2004

[5] SPERO e-questionnaires (http://www.image.ntua.gr/questspero)

[6] SPERO e-survey (http://www.image.ntua.gr/statspero)

[7] SPERO profiles (http://www.image.ntua.gr/questspero/profiles) 